\title{
Percepción a la variabilidad y cambio climático en comunidades campesinas del Valle del Mantaro
}

\section{Variability and climate change perception on the Mantaro Valley rural communities}

\author{
Arturo Anco Arroyo \\ Universidad Continental \\ arturo.anco@yahoo.com
}

\author{
José Miguel Castañeda Cahuana ${ }^{2}$ \\ Universidad Continental \\ jcastanedacahuana@gmail.com
}

\author{
Guillermo Miguel Carlos Gómez ${ }^{3}$ \\ Universidad Continental \\ carlos.gomez.miguel@gmail.com
}

\section{RESUMEN}

El objetivo fue evaluar la percepción a la variabilidad y cambio climático de las comunidades campesinas de Achipampa y Quichuay y comparar con las tendencias y cambios experimentados por la precipitación y temperatura registradas por estaciones meteorológicas cercanas. El análisis estadístico de los registros de precipitación (1964 2014) y temperatura (2009 - 2014) de las estaciones meteorológicas de Ingenio y Laive fue mediante el test de Mann-Kendall y pruebas de homogeneidad, y se contrastó con datos cualitativos colectados a través de una encuesta aplicada a 89 comuneros. Los resultados establecen que la precipitación no presenta tendencia de incremento o reducción en ambas comunidades; en tanto para la temperatura media, mínima y máxima, no existen tendencias claras, salvo el caso de la temperatura máxima en la estación de Laive que muestra una tendencia de disminución. Los comuneros encuestados en Achipampa y Quichuay percibieron que el clima cambió en los últimos cuatro años y que la precipitación y temperatura se incrementaron. La percepción de los comuneros y las estadísticas calculadas sobre las precipitaciones y temperaturas divergen, lo cual no significa que los comuneros estén equivocados, lo más probable es que sean efectos de otros factores meteorológicos y sociales no evaluados por esta investigación. En conclusión, las comunidades campesinas percibieron cambios en el clima mientras que los registros de precipitación y temperatura no presentaron tendencias claras de incremento, disminución o cambio significativo.

\section{ABSTRACT}

The objective was to evaluate the variability and climate change perception of Quichuay and Achipampa rural communities and to compare with trends and experienced changes by recorded precipitation and temperature by weather stations nearby. The statistical analysis of rainfall records (1964 - 2014) and temperature (2009 - 2014) from the Ingenio and Laive Weather Stations was performed by the Mann-Kendall and homogeneity tests, and contrasted with qualitative data collected through an 89 community members' survey. The results establishes no precipitation increasing or decreasing trends in both communities; while for the average, minimum and maximum temperature, there are no clear trends except in the case of the maximum temperature in the Laive station showing a decreasing trend. The Achipampa and Quichuay respondent commoners perceived that the climate changed in the past four years and that the precipitation and temperature increased. The commoners' perception and calculated statistics about precipitations and temperatures diverge, which does not mean that commoners are wrong, probably there are other meteorological and social factors effects don't evaluated by this research. In conclusion, the rural communities perceived changes in climate while precipitation and temperature records showed no clear increase, decrease or significant change trends.

Keywords: Perception, climate change, precipitation, temperature.

Palabras clave: Percepción, cambio climático, precipitación, temperatura.

Historial del artículo:

Recibido: 11 de octubre de 2015. Aprobado: 17 de noviembre de 2015. Disponible en línea: 30 de diciembre de 2015

1 Ingeniero forestal y ambiental, consultor forestal y ambiental.

2 Ingeniero forestal y ambiental, consultor en seguridad y medio ambiente.

3 Ingeniero forestal y ambiental, becario del programa Iniciativas de Conservación de la Amazonía Andina-ICAA. 


\section{INTRODUCCIÓN}

Esta investigación desarrolla el tema de las percepciones de las comunidades campesinas frente al cambio climático, que constituye uno de los principales desafíos ambientales de nuestro tiempo (1). Sus consecuencias adversas ya son evidentes a escala mundial, así se tienen: las modificaciones en los patrones de precipitación y temperatura, que vienen afectando significativamente la disponibilidad de agua para el consumo humano y la agricultura e incremento de frecuencia, duración e intensidad de diversos fenómenos naturales alrededor del mundo $(2,3)$.

El Perú, de acuerdo con los indicadores de riesgo climático desarrollados por el Centro Tyndall, es uno de los países más vulnerables frente al cambio climático, se encuentra entre los 10 países con mayor número de personas afectadas desde 1991 hasta el 2000, debido propias concepciones, relaciones, percepciones sobre la naturaleza y sus territorios, al igual que sobre la historia de los cambios ambientales, incluidos los relacionados al clima (8).

La percepción implica y representa un proceso dialectico de construcción de conocimiento local, a partir de la experiencia vivida. La relación entre el ser humano y su ambiente es, en parte, el reflejo de las percepciones en un contexto determinado, donde a la vez el hombre construye su espacio e implementa mecanismos de supervivencia (9).

En tal sentido, la percepción de los pobladores con respecto al cambio climático complementa a los registros meteorológicos históricos y permite entender comportamientos y grados de precaución que los individuos consideran para hacer frente a las modificaciones climáticas (10), sirviendo de base para

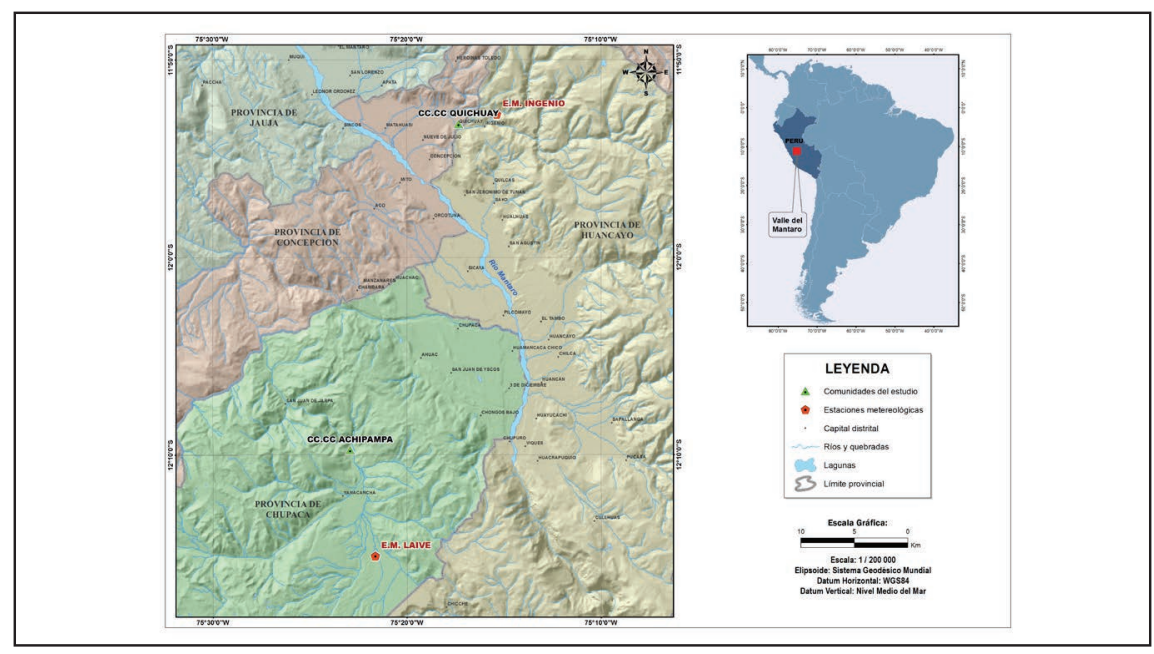

Figura $\mathrm{N}^{\circ}$ 1: Ubicación de comunidades campesinas y estaciones meteorológicas.

al incremento en los eventos climáticos extremos, la alta dependencia del clima en actividades primarias como la agricultura y una reducida capacidad institucional para ejecutar medidas de adaptación (4).

El clima es variable y en muchas partes del mundo también extremo, situación que posibilitó el desarrollo socioeconómico en mayor o menor medida de diversas poblaciones rurales de agricultores, recolectores, pescadores y pastores, quienes ya vienen enfrentándose a los efectos del cambio climático por la alta vulnerabilidad de sus medios de vida sustentados principalmente en recursos naturales $(5,6)$.

Desde la perspectiva de las ciencias sociales, el clima es ante todo el resultado de la forma en que los individuos perciben, se apropian e interpretan los eventos meteorológicos y climáticos que ocurren a su alrededor (7). Es así que cada cultura tiene sus diseñar estrategias de adaptación que contemplen tanto el registro sofisticado de información climática y la realidad local experimentada por la población.

El objetivo del estudio fue la evaluación de la percepción sobre variabilidad y cambio climático en dos comunidades campesinas del Valle del Mantaro y la comparación con las tendencias y cambios de precipitación y temperatura, con fines de evaluar las concordancias entre estas dos fuentes de información.

\section{MATERIAL Y MÉTODOS}

La investigación tiene un diseño descriptivocomparativo debido a que describe las percepciones de los comuneros sobre la variabilidad climática y compara con las tendencias y cambios que experimentó la precipitación y las temperaturas registradas por 
dos estaciones meteorológicas cercanas a estas comunidades.

\section{Área de estudio}

El área de estudio se localiza en las comunidades campesinas de Achipampa y Quichuay, en los distritos de Yanacancha (provincia de Chupaca) y Quichuay (provincia de Huancayo), las mismas que se encuentran cercanas a las estaciones meteorológicas Laive (3990 m s.n.m.) e Ingenio (3 422 m s.n.m.), administradas por el Servicio Nacional de Meteorología e Hidrología (SENAMHI) (figura 1).

\section{Análisis de tendencias de precipitación y temperatura}

Se trabajó con datos de precipitación total mensual registrada para el período 1964 al 2014, y con registros mensuales de temperatura máxima, mínima

\section{Percepción sobre la variabilidad y cambio climático}

Se diseñó una encuesta basada en revisión de literatura sobre percepción de cambio climático $(17,18,19)$, y ajustada a la realidad local de las comunidades estudiadas.

La encuesta aplicada fue estructurada en cinco módulos, 1) datos generales del encuestado; 2) cambios ocurridos en el tiempo y clima; 3) fenómenos meteorológicos comunes y eventos extremos; 4) efectos locales del cambio climático, y 5) conocimiento sobre cambio climático, organizados en un total de 31 preguntas.

Se encuestó un total de 89 comuneros: 45 en Achipampa (figura 2) y 44 en Quichuay, limitando la aplicación solo a comuneros activos con un mínimo de cinco años de residencia dada en estas comunidades

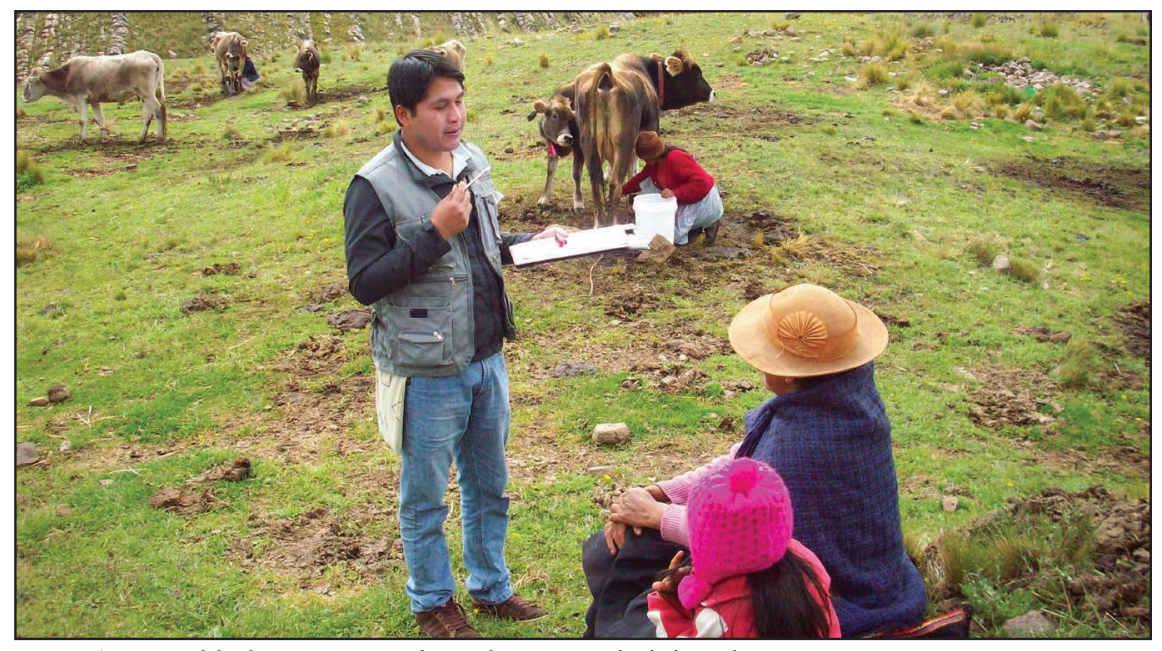

Figura $\mathrm{N}^{\circ}$ 2: Pobladora encuestada en la comunidad de Achipampa.

y media, disponible para el período 2009 al 2014.

Para identificar las tendencias de la serie temporal tanto de precipitación como temperaturas, se empleó el test de Mann-Kendall, que es muy usado en climatología $(11,12)$. Las principales ventajas del uso de esta metodología es que es un método no-paramétrico y por lo tanto no requiere que los datos tengan distribución normal, y que tiene baja sensibilidad a los cambios bruscos debido a datos no homogéneos (12). De acuerdo con este método, la hipótesis nula $\mathrm{HO}$ asume que no hay tendencia y esta es analizada contra la hipótesis alternativa $\mathrm{Hi}$, la cual asume que sí existe tendencia $(13,14)$.

Para detectar puntos de cambio en la media de los valores de precipitación y temperatura, se utilizaron las pruebas de homogeneidad de Pettitt, homogeneidad normal estándar (SNHT) (15), y Buishand (16). una alta presencia de población foránea con menos tiempo de residencia.

El encuestado se realizó durante marzo y abril, y tuvo una duración de 25 a 30 min. Luego se sistematizó las encuestas aplicadas y se analizaron mediante estadística descriptiva.

Finalmente, se elaboró una matriz de comparación, con el fin de analizar, comparar e identificar las coincidencias o divergencias entre los cambios percibidos por las comunidades respecto al clima con los resultados del análisis estadístico de series temporales para precipitación y temperatura. 


\section{RESULTADOS}

\section{Análisis de tendencias de precipitación y temperatura}

El análisis gráfico de la serie temporal de precipitación total anual para el período 1964-2014, muestra la tendencia de incremento en el caso de la estación Laive y de disminución para la estación de Ingenio (figura 3). Sin embargo, estadísticamente empleando el test de Mann-Kendall, se evidencia que no existen tendencias de precipitación para ambas estaciones, puesto que el valor-p calculado es mayor que el nivel
De acuerdo con el análisis de tendencias mediante la prueba de Mann-Kendall para la temperatura máxima, mínima y media mensual para la serie temporal del 2009 al 2014, se encontró que solo la temperatura máxima registrada por la estación Laive presenta una tendencia de disminución, dado que el valor - $p$ computado es menor que el nivel de significación alfa $=0,05$ para esta variable (tabla 3).

Las pruebas de homogeneidad a la serie temporal de temperaturas para ambas estaciones evidenciaron que solo la temperatura máxima mensual en la estación Laive e Ingenio, presentó un período de modificación de los valores medios, puesto que el valor $-p$

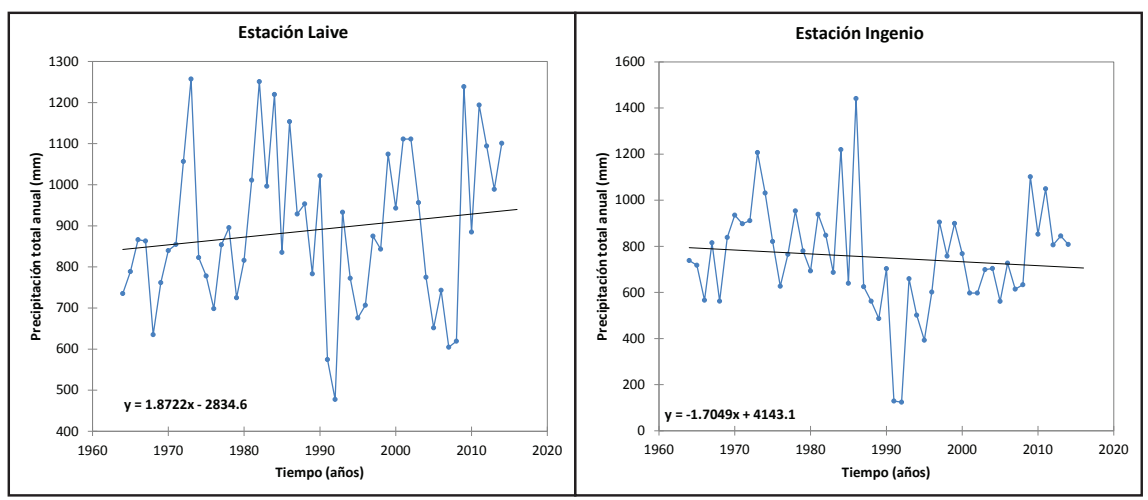

Figura $\mathrm{N}^{\circ}$ 3: Precipitación total anual para las estaciones de Laive e Ingenio.

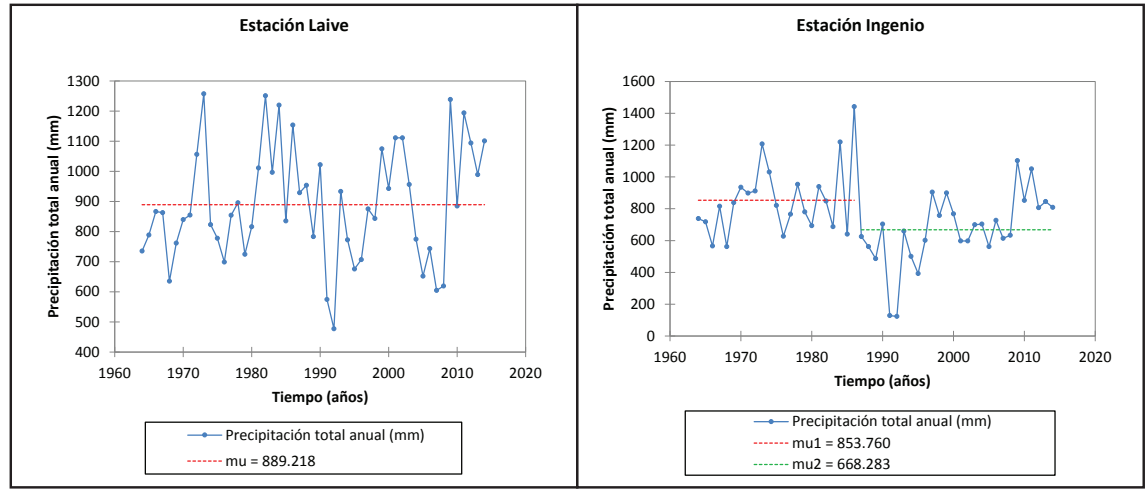

Figura $\mathrm{N}^{\circ}$ 4: Cambios en la precipitación total mensual.

de significación alfa $=0,05$ (tabla 1).

Asimismo, las diferentes pruebas de homogeneidad aplicada a la serie temporal de precipitación total anual, muestran para el caso de la estación Laive que los datos son homogéneos y no existe una fecha en la que hay un cambio en los datos medios. Esta situación sí se presentó en el caso de la estación de Ingenio, puesto que el valor - $p$ computado es menor que el nivel de significación alfa $=0,05$ (tabla 2), donde a partir del año 1987 se presentó una reducción en la media de la precipitación total anual, pasando de 853 $\mathrm{mm}$ a $668 \mathrm{~mm}$ al año (figura 4). computado es menor que el nivel de significación alfa $=0,05$ (tabla 4), es decir, una fecha en la cual cambiaron los datos.

Para el caso de la estación Laive e Ingenio, la temperatura máxima media mensual cambió a partir de noviembre del 2010, experimentando disminuciones entre $1,1^{\circ} \mathrm{C}$ y $2{ }^{\circ} \mathrm{C}$ (figura 5).

\section{Características generales de las comunidades}

La comunidad de Achipampa concentra una población 
Tabla N ${ }^{\circ}$ 1: Test de Mann-Kendall para precipitación total anual (período 1964-2014).

\begin{tabular}{lrr}
\hline & \multicolumn{1}{c}{$\begin{array}{c}\text { Estación } \\
\text { Laive }\end{array}$} & $\begin{array}{c}\text { Estación } \\
\text { Ingenio }\end{array}$ \\
\hline Tau de Kendall & 0,107 & $-0,067$ \\
$\mathrm{~S}$ & 136,000 & $-86,000$ \\
$\operatorname{Var}(\mathrm{S})$ & 15157,333 & 15157,333 \\
Valor -p (bilateral) & 0,273 & 0,490 \\
alfa & 0,05 & 0,05 \\
\hline
\end{tabular}

Tabla № 2: Test de homogeneidad Mann-Kendall para precipitación total anual (período 1964-2014).

\begin{tabular}{lrc}
\hline \multicolumn{1}{c}{ Prueba } & $\begin{array}{c}\text { Estación Laive } \\
\text { (Valor }-\mathrm{p} \text { ) }\end{array}$ & $\begin{array}{c}\text { Estación Ingenio } \\
\text { (Valor }-\mathrm{p} \text { ) }\end{array}$ \\
\hline Pettitt & 0,3604 & 0,0279 \\
$\begin{array}{l}\text { Homogeneidad normal } \\
\text { estándar (SNHT) }\end{array}$ & 0,09 & 0,1055 \\
Buishand & 0,3428 & 0,0196 \\
\hline
\end{tabular}

Tabla №3: Test de Mann-Kendall para temperatura máxima, mínima y media mensual.

\begin{tabular}{lrrrrrr}
\hline & \multicolumn{3}{c}{ Estación Laive } & & Estación Ingenio \\
\hline & $\begin{array}{c}\text { Temperatura } \\
\text { máxima }\end{array}$ & $\begin{array}{c}\text { Temperatura } \\
\text { media }\end{array}$ & $\begin{array}{c}\text { Temperatura } \\
\text { mínima }\end{array}$ & $\begin{array}{c}\text { Temperatura } \\
\text { máxima }\end{array}$ & $\begin{array}{c}\text { Temperatura } \\
\text { media }\end{array}$ & $\begin{array}{c}\text { Temperatura } \\
\text { mínima }\end{array}$ \\
\hline Tau de Kendall & $-0,288$ & 0,013 & 0,094 & $-0,095$ & $-0,068$ & 0,009 \\
S & $-728,000$ & 34,000 & 240,000 & $-184,000$ & $-131,000$ & 18,000 \\
Var(S) & 42233,333 & 42297,000 & 42295,333 & 28371,333 & 28393,667 & 28403,333 \\
Valor -p (bilateral) & 0,000 & 0,873 & 0,245 & 0,277 & 0,440 & 0,920 \\
alfa & 0,050 & 0,050 & 0,050 & 0,050 & 0,050 & 0,050 \\
\hline
\end{tabular}

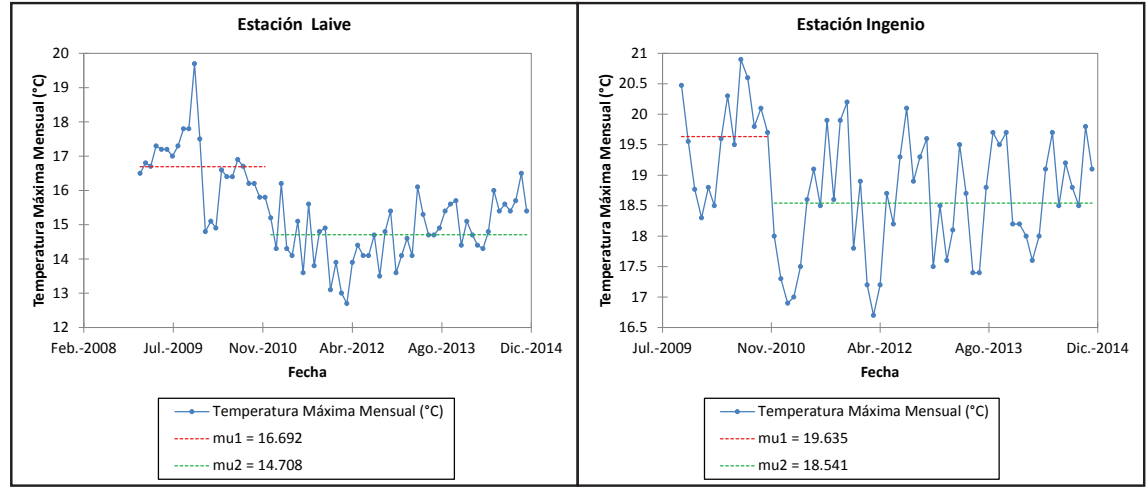

Figura $\mathrm{N}^{\circ}$ 5: Cambios en la temperatura máxima mensual.

de 20 a 40 años (55\%); en tanto que Quichuay, de 51 a 60 años y de 71 a 80 años (40\%).

Las principales actividades económicas en la comunidad de Achipampa son la agricultura y la ganadería (79\%), y de Quichuay, la agricultura y el comercio (66\%).

El $91 \%$ de los comuneros de Achipampa residen más de 11 años en su comunidad, mientras que en Quichuay similar tiempo de residencia lo tiene el $84 \%$.

\section{Percepción sobre la variabilidad y cambio climático}

El $90 \%$ de los encuestados en Achipampa afirman que el clima ha cambiado, en tanto en Quichuay afirman

Tabla № 4: Test de homogeneidad para temperatura máxima, mínima y media mensual.

\begin{tabular}{lcccccc}
\hline & \multicolumn{3}{c}{ Estación Laive } & & \multicolumn{2}{c}{ Estación Ingenio } \\
\hline \multicolumn{1}{c}{ Prueba } & $\begin{array}{c}\text { Temperatura } \\
\text { máxima }\end{array}$ & $\begin{array}{c}\text { Temperatura } \\
\text { media }\end{array}$ & $\begin{array}{c}\text { Temperatura } \\
\text { mínima }\end{array}$ & $\begin{array}{c}\text { Temperatura } \\
\text { máxima }\end{array}$ & $\begin{array}{c}\text { Temperatura } \\
\text { media }\end{array}$ & $\begin{array}{c}\text { Temperatura } \\
\text { mínima }\end{array}$ \\
\hline $\begin{array}{l}\text { Pettitt } \\
\text { Homogeneidad }\end{array}$ & $<0,0001$ & 0,4726 & 0,0973 & 0,0214 & 0,1862 & 0,4889 \\
$\begin{array}{l}\text { normal estándar } \\
\text { (SNHT) }\end{array}$ & $<0,0001$ & 0,8224 & 0,0733 & 0,0038 & 0,065 & 0,3136 \\
\begin{tabular}{l} 
Buishand \\
\hline
\end{tabular} & 0,0001 & 0,5197 & 0,1052 & 0,0126 & 0,2965 & 0,4562 \\
\hline
\end{tabular}


lo mismo el $92 \%$. Los encuestados restantes, en cada caso, mencionaron no haber percibido cambio alguno.

En ambas comunidades se ha percibido modificaciones en precipitación y temperaturas en comparación con años anteriores. El $53 \%$ de los comuneros encuestados en Achipampa aseguran que ahora llueve menos que en años pasados, mientras que en Quichuay el $77 \%$ afirman que hoy llueve más (figura 6).

De manera similar se encontró para el caso de temperaturas máximas y mínimas, que en Achipampa y Quichuay el $86 \%$ y $74 \%$ respectivamente, afirmaron
En relación con el período sobre el cual cada una de las comunidades percibió los cambios en el clima y eventos extremos, se muestra en la tabla 6, que ambas comunidades coinciden en un período medio entre 4,4 y 4,6 años, sobre el que afirman que el clima ha cambiado y que entre el mismo período los eventos climáticos extremos se han vuelto más frecuentes e intensos.

La matriz de comparación (tabla 7) muestra que existen divergencias en las variables de precipitación, temperatura máxima, temperatura mínima, temperatura media, mes con mayor temperatura, mes con temperaturas bajas y coincidencias en la duración

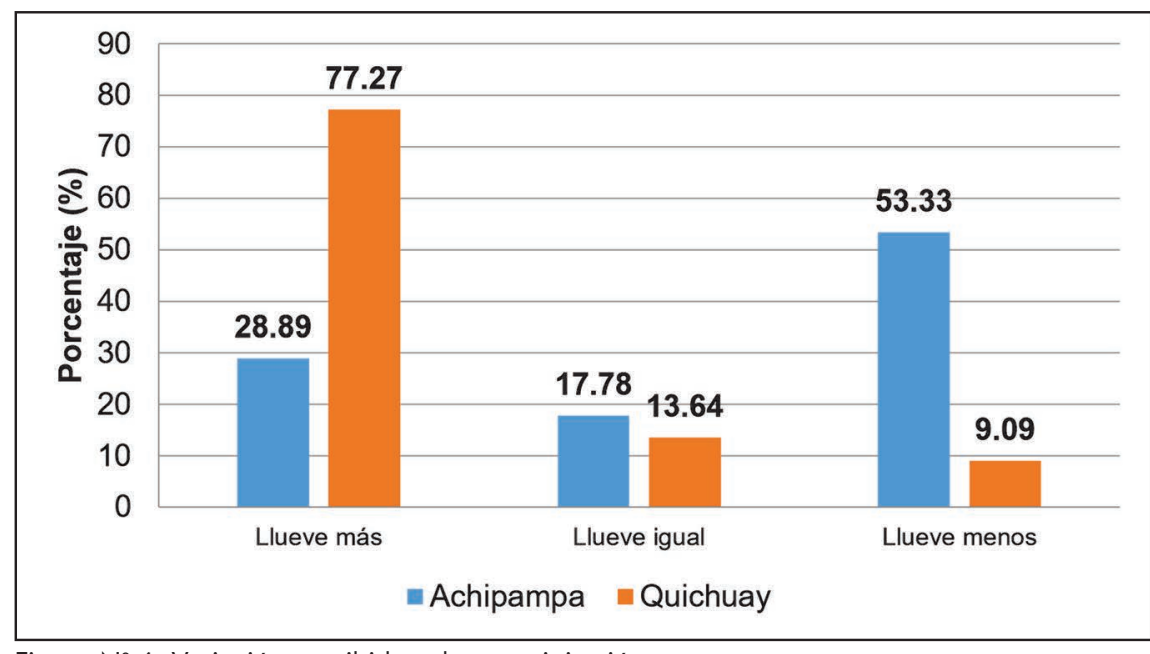

Figura $\mathrm{N}^{\circ}$ 6: Variación percibida sobre precipitación.

Tabla $N^{\circ}$ 5: Intensidad de eventos climáticos.

\begin{tabular}{|c|c|c|c|c|c|c|}
\hline \multirow[b]{2}{*}{$\begin{array}{l}\text { Eventos } \\
\text { climáticos }\end{array}$} & \multicolumn{3}{|c|}{ Achipampa } & \multicolumn{3}{|c|}{ Quichuay } \\
\hline & $\begin{array}{c}\text { Baja } \\
\text { intensidad }\end{array}$ & $\begin{array}{c}\text { Igual } \\
\text { Intensidad }\end{array}$ & $\begin{array}{c}\text { Alta } \\
\text { intensidad }\end{array}$ & $\begin{array}{c}\text { Baja } \\
\text { intensidad }\end{array}$ & $\begin{array}{c}\text { Igual } \\
\text { intensidad }\end{array}$ & $\begin{array}{c}\text { Alta } \\
\text { intensidad }\end{array}$ \\
\hline Lluvias intensas & $26,19 \%$ & $42,86 \%$ & $30,95 \%$ & $17,50 \%$ & $25,00 \%$ & $57,50 \%$ \\
\hline Heladas & $11,63 \%$ & $39,53 \%$ & $48,84 \%$ & $7,69 \%$ & $41,03 \%$ & $51,28 \%$ \\
\hline Tormentas eléctricas & $8,11 \%$ & $35,14 \%$ & $56,76 \%$ & $6,06 \%$ & $45,45 \%$ & $48,48 \%$ \\
\hline
\end{tabular}

que las temperaturas máximas se incrementaron en comparación con años pasados. Asimismo, ambas comunidades percibieron que las temperaturas mínimas disminuyeron en relación con los años anteriores (66 \% en Achipampa y $73 \%$ en Quichuay).

El $75 \%$ de los comuneros de Achipampa mencionaron que el evento climático que actualmente se ha vuelto más frecuente son las lluvias intensas y heladas; en tanto en Quichuay el 78 \% tuvieron igual percepción sobre estos mismos eventos. Respecto a la intensidad de estos eventos en ambas comunidades, se observó que los más intensos son lluvias, heladas y tormentas eléctricas (tabla 5). de la época seca y en el mes de mayor precipitación en la comunidad de Achipampa.

\section{DISCUSIÓN}

Según el test de Mann-Kendall, para el período evaluado entre los años 1964 - 2014 en las estaciones de Laive e Ingenio, la precipitación no presenta tendencia alguna, pese a que la aplicación de tendencia lineal para la serie anual indique un ligero incremento de $18 \mathrm{~mm} /$ década para Laive y una reducción de $17 \mathrm{~mm} /$ década para Ingenio, siendo la última la que más se asemeja a las tendencias reportadas para otras estaciones 
como Huayao (3 308 m s.n.m.) y San Juan de Jarpa (3 726 m s.n.m.), las cuales indican disminución entre 27 y $51 \mathrm{~mm} /$ década, respectivamente (20). Ello refuerza lo planteado por Vuille, quien mencionó que en Los Andes existe poca coherencia espacial entre las tendencias de la precipitación y no existe un patrón claro de incremento o decrecimiento de la misma (21), por lo mismo que las comunidades pueden percibirlos en diferentes momentos, tal es así que en el presente estudio se encontró que los cambios percibidos por con series temporales largas de otras estaciones del Valle del Mantaro, las cuales indican tendencias de incremento (22). Por lo tanto, es necesario profundizar este análisis con datos mensuales de una serie temporal más larga e integrando más estaciones meteorológicas.

En las comunidades de Achipampa y Quichuay el $90 \%$ y el $92 \%$ de los encuestados, respectivamente, percibieron que el clima cambió, lo cual concuerda

Tabla $N^{\circ}$ 6: Períodos en los que se percibieron cambios en el clima.

\begin{tabular}{llcc}
\hline Comunidades & Estadístico & $\begin{array}{c}\text { Tiempo en que } \\
\text { percibió cambios } \\
\text { en el clima (años) }\end{array}$ & $\begin{array}{c}\text { Tiempo en que } \\
\text { percibió cambios en } \\
\text { eventos climáticos } \\
\text { extremos (años) }\end{array}$ \\
\hline Achipampa & Media & 4,330 & 4,000 \\
Quichuay & Desv. estándar & 3,022 & 2,651 \\
Total & Dedia & 5,050 & 4,880 \\
& Nesvándar & 4,032 & 4,767 \\
& Desv. estándar & 4,670 & 4,440 \\
& & 3,541 & 3,858 \\
\hline
\end{tabular}

Tabla № 7: Matriz de comparación de percepción con registro de estaciones.

\begin{tabular}{|c|c|c|c|c|}
\hline Variable & $\begin{array}{c}\text { Estación } \\
\text { Laive }\end{array}$ & $\begin{array}{l}\text { Percepción } \\
\text { Achipampa }\end{array}$ & $\begin{array}{l}\text { Estación } \\
\text { Ingenio }\end{array}$ & $\begin{array}{c}\text { Percepción } \\
\text { Quichuay }\end{array}$ \\
\hline Precipitación & Igual & Disminuyó & Igual & Se incrementó \\
\hline $\begin{array}{l}\text { Duración de la } \\
\text { época seca }\end{array}$ & $\begin{array}{c}5 \text { meses } \\
\text { (Abr-Sep) }\end{array}$ & $\begin{array}{l}5 \text { meses } \\
\text { (Abr-Sep) }\end{array}$ & $\begin{array}{c}5 \text { meses } \\
\text { (Abr-Sep) }\end{array}$ & $\begin{array}{c}5 \text { meses } \\
\text { (Abr-Sep) }\end{array}$ \\
\hline $\begin{array}{l}\text { Temperatura } \\
\text { máxima }\end{array}$ & Disminuyó & $\begin{array}{l}\text { Más calor que } \\
\text { años anteriores }\end{array}$ & Disminuyó & $\begin{array}{l}\text { Más calor que } \\
\text { años anteriores }\end{array}$ \\
\hline $\begin{array}{l}\text { Temperatura } \\
\text { mínima }\end{array}$ & Igual & $\begin{array}{l}\text { Más frío que } \\
\text { años anteriores }\end{array}$ & Igual & $\begin{array}{c}\text { Más frio que } \\
\text { años anteriores }\end{array}$ \\
\hline $\begin{array}{l}\text { Temperatura } \\
\text { media }\end{array}$ & Igual & Se incrementó & Igual & Se incrementó \\
\hline $\begin{array}{l}\text { Mes con mayor } \\
\text { temperatura }\end{array}$ & Noviembre & Agosto & Noviembre & Julio y agosto \\
\hline $\begin{array}{l}\text { Mes con menor } \\
\text { temperatura }\end{array}$ & Julio & Agosto & Julio & Agosto \\
\hline $\begin{array}{l}\text { Mes con mayor } \\
\text { precipitación }\end{array}$ & Febrero & Febrero & Enero & Febrero \\
\hline
\end{tabular}

Achipampa y Quichuay se dieron en los últimos 4,4 a 4,6 años, mientras que en otras comunidades rurales como en Ecuador estos cambios se han percibido en los últimos 8 años (18).

En tanto, para la temperatura media, mínima y máxima, no existen tendencias claras, salvo el caso de la temperatura máxima en la estación Laive que muestra una disminución. Sin embargo, dado que la serie temporal evaluada es corta, limitó la comparación con casos similares reportados en otras zonas rurales del Perú como Piura, Ancash, Cusco y Apurímac $(23,24,25,26)$ y en otros países como Colombia, donde el 89 \% de la población de Santander percibió (18), evidenciándose que el cambio climático está siendo percibida en diversas zonas a nivel nacional e internacional.

El $76 \%$ de la población encuestada coincidió en afirmar que las trasformaciones más evidentes en el clima han 
sido el aumento de la precipitación y la temperatura. Estas opiniones sobre el aumento de la precipitación y la temperatura son comparables con otros estudios realizados en comunidades campesinas del Perú (23, 24, 25, 26), y en países cercanos como Ecuador, donde se encontró que el $75 \%$ de los campesinos encuestados mencionaron que ahora hace más calor que antes y llueve más (27).

La percepción de los comuneros y las estadísticas calculadas sobre las precipitaciones y temperaturas divergen en su mayoría en ambos fenómenos, pero sí coinciden en el tiempo de la estación seca (tabla 7). Esto podría darse porque las comunidades necesitan mayor cantidad de lluvias de las que están disponibles y por otros factores ambientales que vienen ocasionando una menor disponibilidad de lluvias, así como un incremento de calor, sin que ello signifique que los comuneros estén equivocados. Por lo tanto, para futuras investigaciones debe realizarse un análisis más completo, considerando no solo precipitación y temperatura sino otras variables meteorológicas adicionales, así como información social más detallada, dado que existen diversos factores que influyen en la percepción social de las personas (24). Esto refuerza además lo planteado por Berkes y colaboradores, quienes afirman que el conocimiento rural basado en sus subjetividades, experiencias y por ende en sus percepciones conforma otro tipo de conocimiento científico (28), que al parecer es muy complejo para ser comparable con registros de variables meteorológicas específicas.

Finalmente, los comuneros encuestados creen que el cambio climático es un fenómeno que ha venido afectando paulatinamente su cotidianidad y que continúa ocurriendo a nivel global y local, por lo que podemos afirmar que en las comunidades de Achipampa y Quichuay no existe un buen entendimiento sobre dicho fenómeno, situación que influyó en la divergencia de la percepción sobre las variaciones en precipitación y temperatura con las series temporales de las estaciones meteorológicas de Laive e Ingenio.

En conclusión, la precipitación no presenta tendencia de incremento o reducción en ambas comunidades; y para la temperatura media, mínima y máxima, no existe tendencias. Los comuneros encuestados, por su parte, percibieron que el clima cambió en los últimos cuatro años y que la precipitación y temperatura se incrementaron. Las comunidades campesinas percibieron cambios en el clima mientras que los registros de precipitación y temperatura no presentaron tendencias claras de incremento, disminución o cambio significativo

\section{Agradecimientos}

A la Universidad Continental por el apoyo para la realización del estudio; también a los encuestadores.

\section{REFERENCIAS BIBLIOGRÁFICAS}

1. World Bank Group. Climate change. In Group WB. Wolrd Development Report 2015: Mind, society and behavior. Washington; 2015.

2. Intergovernmental Panel on Climate Change. Summary for policymakers. In Field CB, Barros VR, Dokken DJ, Mach KJ, Mastrandrea MD, Bilir $T E$, et al., editors. Climate change 2014: Impacts, adaptation, and vulnerability. Part A: Global and sectoral aspects. Cambridge: Cambridge University Press; 2014.

3. Trenberth KE, Dai A, Rasmussen RM, Parsons DB. The Changing character of precipitation. Bull. Amer. Meteor. Soc. 2003; 84: p. 1205-1217.

4. Brooks N, Adger N. Country level risk measures of climate-related natural disasters and implications for adaptation to climate change. UK: University of East Anglia, Centre for Climate Change Research; 2003.

5. Nakashima D, Galloway K, Thulstrup H, Ramos A, Rubis J. Weathering Uncertainty: Traditional Knowledge for Climate Change Assessment and Adaptation París: UNESCO-UNU; 2012.

6. Ulloa A, Escobar EM, Donato LM, Escobar P. Mujeres indigenas y cambio climatico, perpectivas latinoamericanas Bogotá: Fundación Natura. UNODC.UNAL; 2008.

7. Mariño N. Reflexiones sobre la perspectiva cultural en las politicas de cambio climatico en Colombia: un acercamiento al analisis cultural y espacial de las politicas publicas. In Ulloa A. Perspectivas culturales del clima. Bogotá: Universidad Nacional de Colombia; 2011 . p. 575.

8. Heyd T. Climate Change, Individual Responsibilites and Cultural Frameworks. Research in Human Ecology. 2010; 17(2): p. 86-95.

9. Padilla y Sotelo S, Luna A. Percepción y conocimiento ambiental en la costa de Quintana Roo: una caracterización a través de encuestas. Investigaciones Geográficas. 2003;(52): p. 99116.

10. Max-Neef M, Elizalde A, Hopenhayn M. Desarrollo a escala humana, opciones para el futuro. 2 nd ed. Mdrid: Biblioteca CF+S; 2010.

11. Chaouche K, Neppel L, Dieulin C, Pujol N, Ladouche B, Martin E, et al. Analyses of precipitation, temperature and evapotranspiration in a French Mediterranean region in the context of climate change. Geoscience. 2010; 342(3): p. 234-243. 
12. De La Mora C, Flores H, Ruíz J, Chávez Á, Figueroa A. Impacto del cambio climático en las tendencias de la evaporación en la presa La Vega, Teuchitlán, Jalisco, México. Revista Mexicana de Ciencias Agrícolas. 2014;(10): p. 1993-2005.

13. Ehsanzadeh E, Saley H, Ouarda T, Burn D, Pietroniro A, Seidou O, et al. Analysis of changes in the Great Lakes hydro-climatic variables. Journal of Great Lakes Research. 2013; 39(3): p. 383-394.

14. Urrutia J, Palomino R, Salazar H. Metodología para la imputación de datos faltantes en meteorología. Scientia ef Technica. 2010; 17(46): p. 44-49.

15. Alexandersson $\mathrm{H}$, Moberg A. Homogenization of Swedish temperature data. International Journal of Climatology. 1997; 17(35-34): p. 25-34.

16. Buishand T. Some methods for testin the homogeneity of rainfall records. Journal of Hydrology. 1982; 58: p. 11-27.

17. Velarde L. Evaluación de la percepción y los factores determinantes en la implementación de medidas de adaptación al cambio y variabilidad climática por los productores de leche de la cuenca del río La Villa, Panamá. Tesis de postgrado. Turrialba: Centro Agronómico Tropical de Investigación y Enseñanza - CATIE, Escuela de Posgrado; 2012.

18. Pinilla M, Rueda A, Pinzón C, Sánchez J. Percepciones sobre los fenómenos de variabilidad climática y cambio climático entre campesinos del centro de Santander, Colombia. Ambiente y Desarrollo. 2012; 16(31): p. 25-37.

19. Vergara K. Variabilidad climática percepción ambiental y estrategias de adaptación de la comunidad campesina de Conchucos, Ancash. Tesis de pregrado. Lima: Pontificia Universidad Católica del Perú, Facultad de Letras y Ciencias Humanas; 2011.

20. Avalos G, Cubas F, Oria C, Díaz A, Quispe N,
Rosas $G$, et al. Atlas climático de precipitación y temperatura del aire en la cuenca del río Mantaro Lima: Servicio Nacional de Meteorología e Hidrología - SENAMHI; 2010.

21. Vuille M, Bradley R, Werner M, Keimig F. 20th century climate change in the tropical Andes observations and model results. Climate change. 2003;(59): p. 75-99.

22. Silva Y, Trasmonte G. Tendencias en las lluvias y temperaturas del valle del Mantaro. In Villaverde $M$, editor. Eventos meteorológicos extremos (sequías, heladas y lluvias intensas) en el valle del Mantaro. Lima: Instituto Geofísico del Perú; 2012. p. 60-62.

23. De La Torre C, Cruz M, Valdivia G. Los desafíos de la adaptación al cambio climático en comunidades rurales altoandinas Liima: Soluciones Prácticas ITDG; 2012.

24. Gómez C, Prado G, Carrasco H, Ferradas P, Carbonel D, Monroe J. Tecnologías frente a la variabilidad climática Lima: Soluciones Prácticas ITDG; 2011.

25. Torres J, Gómez A. Adaptación al cambio climático: de los fríos y los calores en los Andes Torres J, Gómez A, editors. Lima: Soluciones Prácticas - ITDG; 2008.

26. Torres J, Gómez A, Berrú M. Gestión de cuencas para enfrentar el cambio climático y el Fenómeno El Niño Torres J, Gómez A, Berrú M, editors. Lima: Soluciones Prácticas - ITDG; 2008.

27. VanderMolen K. Percepciones de cambio climático y estrategias de adaptación en las comunidades agrícolas de Cotacachi. Revista Ecuador Debate. 2011 ;(82): p. 145-157.

28. Berkes F, Colding J, Folke C. Rediscovery of traditional ecological knowledge as adaptive management. Ecologial Applications. 2000; 10(5): p. 1251-1262. 